\title{
Interactive comment on "Detecting layer height of smoke aerosols over vegetated land and water surfaces via oxygen absorption bands: Hourly results from EPIC/DSCOVR satellite in deep space” by Xiaoguang Xu et al.
}

\section{Xu (Referee)}

fengxu@jpl.nasa.gov

Received and published: 23 January 2019

In this paper, Xu et al. extends their earlier work on aerosol layer height (ALH) and aerosol optical depth (AOD) retrieval from ocean to land - using EPIC observations. Comparison to CALIOP retrieval of ALH and AERONET product of AOD show an error of $0.58 \mathrm{~km}$ in retrieved ALH and an error of 0.05 in retrieved AOD, respectively. This work was very well organized. I don't have questions on the general technical routines presented in this work as the previous publications by the authors along this line of research have laid down a solid basis for proceeding with this work. My comments 
below are for the authors to somehow clarify their approach that readers can better digest the ideas behind it: 1 . What is source of oxygen profile adopted in EPIC ALH and AOD retrievals? Is there any impact from temperature on oxygen profile and then on ALH retrieval ? 2. The sensitivity of O2-A and $\mathrm{B}$ bands to the profiles of smoke aerosols are clear from Figure 3. I'm curious how much the sensitivity will change if aerosol absorption changes gradually from being strongly absorbing to being weakly absorbing ? 3. I believe the authors have published it elsewhere, but it would be helpful to some readers if some comments from authors' side can be made on the sensitivity of O2-A and B bands to the width of aerosol layer. 4. To give people a better idea about the "real" sensitivity that measurements have to AOD and ALH, it will be nicer if the authors can describe in the caption of Figure 3 EPIC's measurement errors in the two $\mathrm{O} 2$ bands. Probably it would be clear if Z-score (ratio of difference of signals in A/B band signals as normalized by measurement errors) is plotted as its axis. 5. Page 5, though there is a reason (geolocation) on the aggregation of pixels into a box of $3 \times 3$ pixels, do the authors think the price to pay (reduction of spatial resolution from $24 \mathrm{~km}$ to $8 \mathrm{~km}$ ) too high. What if retrieval is directly implemented on 1 by $1 \mathrm{grid}$ to retain EPIC's original $8 \mathrm{~km}$ resolution? 6 . Page 8 , the smoke particle properties are described for retrieving $A O D$ and $A L H$. It might helpful if some comments can be given regarding if the pre-determined aerosol model have certain errors and its potential impact on ALH and $A O D$ retrieval accuracy.

Some suggested editorial changes: 1 . The reference for Lewis [1994] is not complete. Second author's last name is missing. Correct the citation of this reference in line 24 of Page 6 as well. 2. Delete the redundant set of words "over land" in line 26 of Page 8. 3. Line 27 of Page 8: should "separated" be "separate"?

Interactive comment on Atmos. Meas. Tech. Discuss., doi:10.5194/amt-2018-414, 2018.

Interactive comment 